UCI-TR-2012-08

TUM-HEP 845/12

DESY-12-108

FLAVOUR(267104)-ERC-19

\title{
The $\mu$ term and neutrino masses
}

\author{
Mu-Chun Chen ${ }^{1}{ }^{a}$, Michael Rat $2^{2} b$, Christian Staudt $3^{3}$, Patrick \\ K.S. Vaudrevange $4 c$ \\ ${ }^{a}$ Department of Physics and Astronomy, University of California, \\ Irvine, California 92697-4575, USA \\ ${ }^{b}$ Physik-Department T30, Technische Universität München, \\ James-Franck-Straße, 85748 Garching, Germany \\ ${ }^{c}$ Deutsches Elektronen-Synchrotron DESY, Notkestraße 85, 22607 Hamburg, Germany
}

\begin{abstract}
The well-known Giudice-Masiero mechanism explains the presence of a $\mu$ term of the order of the gravitino mass, but does not explain why the holomorphic mass term is absent in the superpotential. We discuss anomaly-free discrete symmetries which are both compatible with $\mathrm{SU}(5)$ unification of matter and the GiudiceMasiero mechanism, i.e. forbid the $\mu$ term in the superpotential while allowing the necessary Kähler potential term. We find that these are $\mathbb{Z}_{M}^{R}$ symmetries with the following properties: (i) $M$ is a multiple of four; (ii) the Higgs bilinear $H_{u} H_{d}$ transforms trivially; (iii) the superspace coordinate $\theta$ has charge $M / 4$ and, accordingly, the superpotential has charge $M / 2$; (iv) dimension five proton decay operators are automatically absent. All $\mathbb{Z}_{M}^{R}$ symmetries are anomaly-free due to a non-trivial transformation of a Green-Schwarz axion, and, as a consequence, a holomorphic $\mu$ term appears at the non-perturbative level. There is a unique symmetry that is consistent with the Weinberg operator while there is a class of $\mathbb{Z}_{M}^{R}$ symmetries which explain suppressed Dirac neutrino masses.
\end{abstract}

\footnotetext{
${ }^{1}$ Email: muchunc@uci.edu

${ }^{2}$ Email: michael.ratz@tum.de

${ }^{3}$ Email: christian.staudt@tum.de

${ }^{4}$ Email: patrick.vaudrevange@desy.de
} 


\section{Motivation}

The minimal supersymmetric standard model (MSSM) is a very appealing extension of the standard model of particle physics. Supersymmetry promises to stabilize the electroweak scale against radiative corrections. The structure of matter hints at unification, and the attractive picture of precision gauge unification [1] enabled by supersymmetry introduces the scale of grand unification $M_{\mathrm{GUT}}=\mathrm{a}$ few $\times 10^{16} \mathrm{GeV}$. The MSSM also provides a compelling dark matter candidate.

On the other hand, the MSSM has various problems. Usually the MSSM comes with matter or $R$ parity [2, 3] which eliminates the most troublesome baryon number violating interactions, and ensures the stability of the aforementioned dark matter particle. Yet, even after imposing matter parity, there are certain serious shortcomings. One of them is the so-called " $\mu$ problem" which consists in the question why the holomorphic mass term for the Higgs bilinear is of the order of the electroweak scale. In addition, there is the dimension five proton decay problem [3] 5 ] (cf. also [6]).

It is hence clear that the MSSM requires additional ingredients beyond matter parity. In this study we analyze anomaly-free discrete symmetries which forbid the $\mu$ term. As we shall demonstrate, requiring that the symmetries be compatible with the GiudiceMasiero solution [7] to the $\mu$ problem and SU(5) leads to very restricted classes of solutions, depending on whether neutrinos are Majorana or Dirac particles. In the first case, the solution is unique and even compatible with $\mathrm{SO}(10)$ while in the second case the smallness of the Dirac neutrino Yukawa coupling can be related to the suppression of the $\mu$ parameter.

\section{Naturally suppressed $\mu$ term and Dirac neutrino Yukawa couplings from anomaly-free symmetries}

We start by reviewing the explanations of a suppressed $\mu$ term through Kähler potential terms in Section 2.1. Next, we discuss anomaly constraints in Section 2.2. In Section 2.3 we comment on proton decay operators and study settings with $\mathrm{SO}(10)$ relations in Section 2.4. Then, we discuss the appearance of a suppressed holomorphic $\mu$ term and Dirac Yukawa couplings in Section 2.5 and Section 2.6, respectively, and give a short recap in Section 2.7

\subsection{Giudice-Masiero mechanism}

The famous Giudice-Masiero mechanism [7] provides a solution to the $\mu$ problem in the MSSM. Giudice and Masiero pointed out that in supergravity an effective holomorphic $H_{u} H_{d}$ bilinear, i.e. an effective $\mu$ term, can arise from the (non-holomorphic) Kähler potential term

$$
K \supset k_{H_{u} H_{d}} \frac{X^{\dagger}}{M_{\mathrm{P}}} H_{u} H_{d}+\text { h.c. . }
$$


Here $X$ is the (spurion) field that breaks supersymmetry and $k_{H_{u} H_{d}}$ and $M_{\mathrm{P}}$ denote a coefficient and the Planck scale, respectively. Inserting the $F$ term vacuum expectation value (VEV) $F_{X}$ of $X$ leads to an effective superpotential term

$$
\mathscr{W}_{\text {eff }} \sim \frac{F_{X}}{M_{\mathrm{P}}} H_{u} H_{d}=: \mu_{\mathrm{eff}} H_{u} H_{d},
$$

with $\mu_{\text {eff }}$ of the order of the gravitino mass $m_{3 / 2}$, which sets the size of soft superpartner masses in gravity mediation.

However, for the Giudice-Masiero mechanism to work, the holomorphic superpotential term $\mu H_{u} H_{d}$ needs to be absent in the first place, or better forbidden by a symmetry. As it turns out, symmetries that can forbid the $\mu$ term are rather constrained. It has been shown [8] that, if one requires the symmetry to be anomaly-free and to commute with $\mathrm{SU}(5)$ (in the matter sector), it has to be an $R$ symmetry (cf. the similar discussion in [9]). As shown by Chamseddine and Dreiner [10], in the MSSM gauged anomaly-free continuous $R$ symmetries are not available. On the other hand, there are strong arguments against global symmetries (cf. [11 for a recent discussion). We are hence led to the conclusion that the symmetry needs to be discrete. In what follows, we therefore will only consider anomaly-free discrete $R$ symmetries. Specifically, we will look at one particular generator which forbids the $\mu$ term. This generator will generate an Abelian discrete $R$ symmetry of order $M$, i.e. a $\mathbb{Z}_{M}^{R}$ symmetry.

One can actually narrow down the potential symmetries even further. Suppose we seek to generate an effective $\mu$ term, Equation (2.2), from the Kähler potential term. Here we assume that $X$ is the field that breaks supersymmetry and generates gaugino masses. Then its $F$ component has to have minus the $R$ charge of the superpotential. One way to see this is by recalling that gaugino masses get induced by the operator $\int \mathrm{d}^{2} \theta X W_{\alpha} W^{\alpha}$ (with $\theta$ and $W_{\alpha}$ denoting the superspace coordinate and the gauge multiplets, respectively). Since the superpotential $R$ charge $q_{\mathscr{W}}$ equals twice the $R$ charge of $\theta, q_{\theta}$, and the lowest components of $W_{\alpha}$ (i.e. the gauginos) carry $R$ charge $q_{\theta}$, the $X$ superfield needs to be inert under the (discrete) $R$ symmetry. Therefore, the Higgs bilinear $H_{u} H_{d}$ needs to be neutral as well. Altogether we have found that an anomalyfree and $\mathrm{SU}(5)$ compatible symmetry that forbids the $\mu$ term in the MSSM has to be discrete, and under this symmetry,

$$
\begin{aligned}
& \theta \rightarrow \mathrm{e}^{2 \pi \mathrm{i} \frac{q_{\theta}}{M}} \theta, \\
& \mathscr{W} \rightarrow \mathrm{e}^{2 \pi \mathrm{i} \frac{q_{\mathscr{W}}}{M}} \mathscr{W} \quad \text { where } q_{\mathscr{W}}=2 q_{\theta}, \\
& X \rightarrow X \text {, } \\
& H_{u} H_{d} \rightarrow H_{u} H_{d} \text {. }
\end{aligned}
$$

Here and throughout this study we normalize the discrete charges to be integer, i.e. $q_{\theta} \in \mathbb{Z}$.

It is immediately clear that such a symmetry allows effective superpotential terms of the form

$$
\mathscr{W} \supset c_{\Omega} \frac{\Omega}{M_{\mathrm{P}}^{2}} H_{u} H_{d}
$$


where $\Omega$ (with $R$ charge $q_{\mathscr{W}}$ ) denotes the superpotential of some 'hidden sector'. As usual, a non-trivial VEV of $\Omega$ is required to cancel the vacuum energy. This VEV will break the $R$ symmetry, but the breaking is hierarchically small, i.e. of the order of the gravitino mass $m_{3 / 2}$ (cf. the discussion in [12]). That means that, apart from the Giudice-Masiero contribution, one would expect to have a holomorphic ('Kim-Nilles type' [13]) contribution to the $\mu$ parameter of the right size.

\subsection{Anomaly constraints}

Up to now we have only used the fact derived in [8] that SU(5)-compatible and anomalyfree non- $R$ symmetries cannot forbid the $\mu$ term. Now we discuss anomaly constraints on $\mathbb{Z}_{M}^{R}$ symmetries. These constraints have been re-derived recently in [8]. However, there only the special case $q_{\theta}=1$ has been considered, which is too strong a requirement. To see this, consider a $\mathbb{Z}_{8}^{R}$ symmetry, for which there are two different non-trivial possibilities for the superspace charge, $q_{\theta}=1$ and $q_{\theta}=2$. At first glance, one may think that one may rewrite the $q_{\theta}=2$ case as a $\mathbb{Z}_{4}^{R} \times \mathbb{Z}_{2}$ symmetry. This is not the case since 2 and 8 are not coprime 1 The generalization of the anomaly coefficients to arbitrary $q_{\theta}$ is straightforward and deferred to appendix A.

After summarizing the relevant anomaly coefficients for the MSSM in Section 2.2.1 we explain in Section 2.2.2 why 'anomaly universality' must be imposed in models in which the SM gauge group is unified into a simple gauge group. We then proceed by verifying the consistency with anomaly matching in Section 2.2 .3 and show that only $R$ symmetries can forbid the $\mu$ term in Section 2.2.4. Finally, we derive constraints on the order $M$ in Section 2.2.5 and comment on the (ir)relevance of the universality of the mixed hypercharge anomaly in Section 2.2.6.

\subsection{1 $\mathbb{Z}_{M}^{R}$ anomaly coefficients in the MSSM}

In the case of the MSSM the anomaly coefficients $A_{3}^{R}:=A_{\mathrm{SU}(3)_{\mathrm{C}}-\mathrm{SU}(3)_{\mathrm{C}}-\mathbb{Z}_{M}^{R}}, A_{2}^{R}:=$ $A_{\mathrm{SU}(2)_{\mathrm{L}}-\mathrm{SU}(2)_{\mathrm{L}}-\mathbb{Z}_{M}^{R}}$ and $A_{1}^{R}:=A_{\mathrm{U}(1)_{Y}-\mathrm{U}(1)_{Y}-\mathbb{Z}_{M}^{R}}$ read

$$
\begin{aligned}
& A_{3}^{R}=\frac{1}{2} \sum_{g=1}^{3}\left(3 q_{10}^{g}+q_{\frac{g}{5}}^{g}\right)-3 q_{\theta}, \\
& A_{2}^{R}=\frac{1}{2} \sum_{g=1}^{3}\left(3 q_{10}^{g}+q_{\frac{g}{5}}^{g}\right)+\frac{1}{2}\left(q_{H_{u}}+q_{H_{d}}\right)-5 q_{\theta}, \\
& A_{1}^{R}=\frac{1}{2} \sum_{g=1}^{3}\left(3 q_{10}^{g}+q_{\frac{9}{5}}^{g}\right)+\frac{3}{5}\left[\frac{1}{2}\left(q_{H_{u}}+q_{H_{d}}\right)-11 q_{\theta}\right] .
\end{aligned}
$$

Here, $q_{\mathbf{1}}^{g}$ and $q \frac{g}{\mathbf{5}}$ denote the $\mathrm{SU}(5)$-universal $R$ charges of the MSSM superfields $\left(Q^{g}, \bar{U}^{g}, \bar{E}^{g}\right)$ and $\left(\bar{D}^{g}, L^{g}\right)$, respectively, and $g$ represents the flavor index. Accordingly, matter

\footnotetext{
${ }^{1} \mathrm{~A}$ simple way of seeing this is to recall that all elements of $\mathbb{Z}_{4}^{R} \times \mathbb{Z}_{2}$ have the property that taking them to the fourth power yields identity, which is obviously not the case for $\mathbb{Z}_{8}^{R}$.
} 
fermions and Higgsinos have charges $q-q_{\theta}$ while gauginos have charge $q_{\theta}$.

\subsubsection{Anomaly universality and discrete Green-Schwarz mechanism}

If the standard model gauge group is to be unified into $\mathrm{SU}(5)$ or $\mathrm{SO}(10)$, a necessary condition for anomaly cancellation is the universality (cf. the discussion in appendix B.3.

$$
A_{3}^{R}=A_{2}^{R}=A_{1}^{R}=\rho \bmod \eta .
$$

Here we introduce

$$
\eta:= \begin{cases}M / 2, & \text { if } M \text { even } \\ M, & \text { if } M \text { odd }\end{cases}
$$

$\rho$ is a constant which indicates whether or not a Green-Schwarz (GS) mechanism [14] is at work. Specifically, $\rho$ is related to the discrete shift of the GS axion (see Equation (B.9) in appendix B.2. $) . \rho=0$ means that the symmetry is anomaly-free in the conventional sense, i.e. without GS mechanism.

At this point, we would like to comment on certain important properties of the Green-Schwarz mechanism and its discrete version as there seems to be some confusion in the literature:

1. Although the GS mechanism plays a prominent role in string theory, it does not rely on strings. In fact, as shown in appendix B.2, it can entirely be understood in (the path integral formulation of) quantum field theory.

2. Unlike in the continuous case, for discrete symmetries the transformation of the axion is only fixed modulo $\eta$. It will be interesting to see whether this ambiguity can be fixed somehow, e.g. in explicit string-derived models.

3. In the continuous case, the axion has to be massless for the shift symmetry to be a symmetry of the Lagrangean. That is, the axion potential needs to be flat. By contrast, in the discrete case the potential is only required to be periodic, i.e. invariant under the discrete shift, Equation (B.9). Therefore the axion may have a non-trivial mass prior to the breakdown of the symmetry. This is, in a way, somewhat surprising as it means that a massive (and bosonic) state can contribute to an anomaly. Of course, in both cases the symmetry will be broken (spontaneously) once the axion $a$ acquires its VEV.

\subsubsection{Anomaly matching}

It is instructive to use 't Hooft anomaly matching [15] (see [16] for discrete anomaly matching) in order to constrain the properties of anomaly-free GUT-compatible $\mathbb{Z}_{M}^{R}$ symmetries. At the $\mathrm{SU}(5)$ level, there is only one anomaly coefficient $A_{\mathrm{SU}(5)^{2}-\mathbb{Z}_{M}^{R}}$, which we can split into three parts,

$$
A_{\mathrm{SU}(5)^{2}-\mathbb{Z}_{M}^{R}}=A_{\mathrm{SU}(5)^{2}-\mathbb{Z}_{M}^{R}}^{\text {matter }}+A_{\mathrm{SU}(5)^{2}-\mathbb{Z}_{M}^{R}}^{\text {extra }}+5 q_{\theta}
$$


The first term contains the contribution of matter and is given by

$$
A_{\mathrm{SU}(5)^{2}-\mathbb{Z}_{M}^{R}}^{\mathrm{mater}}=\frac{1}{2} \sum_{g=1}^{3}\left(3 q_{10}^{g}+q \frac{g}{\mathbf{5}}\right)-6 q_{\theta} .
$$

Here, we used Equation (A.4a with Dynkin indices $\ell(\overline{\mathbf{5}})=\frac{1}{3} \ell(\mathbf{1 0})=\frac{1}{2}$. The second term in (2.8),$A_{\mathrm{SU}(5)^{2}-\mathbb{Z}_{M}^{R}}^{\text {extra }}$, denotes the contributions of additional fields, e.g. the $\mathrm{SM}$ and $\mathrm{SU}(5)$ breaking Higgs. Finally, the last term in (2.8) represents the gaugino contribution for $\mathrm{SU}(5)$. Yet, by considering the $\mathrm{SU}(3)_{\mathrm{C}}$ and $\mathrm{SU}(2)_{\mathrm{L}}$ subgroups of $\mathrm{SU}(5)$, one can introduce two anomaly coefficients $A_{\mathrm{SU}(3)_{\mathrm{C}}^{2}-\mathbb{Z}_{M}^{R}}^{\mathrm{SU}(5)} A_{\mathrm{SU}(2)_{\mathrm{L}}^{2}-\mathbb{Z}_{M}^{R}}^{\mathrm{SU}(5)}$ at the GUT level,

$$
\begin{aligned}
& A_{\mathrm{SU}(3)_{\mathrm{C}}^{2}-\mathbb{Z}_{M}^{R}}^{\mathrm{SU}(5)}=A_{\mathrm{SU}(3)_{\mathrm{C}}^{2}-\mathbb{Z}_{M}^{R}}^{\text {matter }}+A_{\mathrm{SU}(3)_{\mathrm{C}}^{2}-\mathbb{Z}_{M}^{R}}^{\mathrm{extra}}+3 q_{\theta}+\frac{1}{2} \cdot 2 \cdot 2 \cdot q_{\theta}, \\
& A_{\mathrm{SU}(5)_{\mathrm{L}}^{2}-\mathbb{Z}_{M}^{R}}^{\mathrm{SU}}=A_{\mathrm{SU}(2)_{\mathrm{L}}^{2}-\mathbb{Z}_{M}^{R}}^{\mathrm{matter}}+A_{\mathrm{SU}(2)_{\mathrm{L}}^{2}-\mathbb{Z}_{M}^{R}}^{\mathrm{extra}}+2 q_{\theta}+\frac{1}{2} \cdot 2 \cdot 3 \cdot q_{\theta},
\end{aligned}
$$

where we artificially split the gaugino contributions into those from the adjoint representations of $\mathrm{SU}(2)_{\mathrm{L}}$ or $\mathrm{SU}(3)_{\mathrm{C}}$, respectively, and in those coming from the extra gauginos in the $(\mathbf{3}, \mathbf{2})_{-5 / 6} \oplus(\overline{\mathbf{3}}, \mathbf{2})_{5 / 6}$ representation. Assume now there is some (unspecified) mechanism that breaks the GUT symmetry down to the SM symmetry, and thus removes the extra gauginos, while leaving $\mathbb{Z}_{M}^{R}$ unbroken 2 Then, the coefficients

$$
\begin{aligned}
A_{\mathrm{SU}(3)_{\mathrm{C}}^{2}-\mathbb{Z}_{M}^{R}}^{\mathrm{SU}(5) \text { boken }} & =A_{\mathrm{SU}(3)_{\mathrm{C}}^{2}-\mathbb{Z}_{M}^{R}-2 q_{\theta}}^{\mathrm{SU}(5)} \\
A_{\mathrm{SU}(2)_{\mathrm{L}}^{2}-\mathbb{Z}_{M}^{R}}^{\mathrm{SU}(5)} & =A_{\mathrm{SU}(2)_{\mathrm{L}}^{2}-\mathbb{Z}_{M}^{R}}^{\mathrm{SU}}-3 q_{\theta}
\end{aligned}
$$

cannot be equal, i.e. the anomaly coefficients cannot be universal, unless there are split multiplets contributing to $A_{\mathrm{SU}(N)^{2}-\mathbb{Z}_{M}^{R}}^{\text {extra }}$ (where we use $A_{\mathrm{SU}(3)_{\mathrm{C}}^{2}-\mathbb{Z}_{M}^{R}}^{\mathrm{matter}}=A_{\mathrm{SU}(2)_{\mathrm{L}}^{2}-\mathbb{Z}_{M}^{R}}^{\text {matter }}$. That is, 't Hooft anomaly matching for (discrete) $R$ symmetries implies the presence of split multiplets below the GUT scale.

\subsubsection{Only $R$ symmetries can forbid the $\mu$ term}

Given that SM matter furnishes complete SU(5) representations and the attractive picture of MSSM gauge unification, arguably the most plausible candidates for such split multiplets are the Higgs fields. Requiring that the Higgs fields cancel the mismatch of gaugino contributions to the anomalies, we obtain

$$
\frac{1}{2}\left(q_{H_{u}}+q_{H_{d}}-2 q_{\theta}\right)=q_{\theta} \bmod \eta
$$

\footnotetext{
${ }^{2}$ If one is to obtain the exact MSSM spectrum after GUT breaking, this mechanism cannot be spontaneous symmetry breaking in four dimensions [17]. On the other hand, extra dimensions, especially in the framework of heterotic orbifolds, naturally can give discrete $R$ symmetries as remnants of higher dimensional Lorentz symmetry, see e.g. [18.
} 
implying

$$
\begin{aligned}
q_{H_{u}}+q_{H_{d}} & =4 q_{\theta} \bmod 2 \eta \\
& =2 q_{\mathscr{W}} \bmod 2 \eta \\
& \neq q_{\mathscr{W}} \quad \bmod M \quad \text { for } q_{\mathscr{W}} \neq 0 \quad \bmod M .
\end{aligned}
$$

Therefore, non $-R$ symmetries with $q_{\theta}=q_{\mathscr{W}}=0$ cannot forbid the $\mu$ term. But in case of non-trivial $\mathbb{Z}_{M}^{R}$ symmetries (i.e. $M \geq 3$ ) the $\mu$ term will always be forbidden, as it should be, since only chiral contributions can 'repair' the gaugino mismatch.

A remark is in order to show that $\mathbb{Z}_{M}^{R}$ with $q_{\mathscr{W}}=0 \bmod M$ is not an $R$ symmetry. In the case $q_{\mathscr{W}}=0 \bmod M$ we find two solutions for $q_{\theta}$ : either $q_{\theta}=0$, such that the symmetry is clearly non- $R$, or (for $M$ even) $q_{\theta}=M / 2$. However, since the transformation $\theta \mapsto-\theta$ and $\Psi \mapsto-\Psi$ for all fermions $\Psi$ is always a symmetry, one can shift the $\mathbb{Z}_{M}^{R}$ charges by $M / 2$ such that again $q_{\theta}=0$. Hence, $\mathbb{Z}_{M}^{R}$ with $q_{\mathscr{W}}=0 \bmod M$ is equivalent to a non- $R$ symmetry [19].

\subsubsection{Constraints on the order $M$}

Using Equation (2.13) and assuming a Giudice-Masiero-like mechanism such that $q_{H_{u}}+$ $q_{H_{d}}=0 \bmod M$ from (2.3d), we obtain

$$
2 q_{\mathscr{W}}=0 \bmod M,
$$

which implies, given the freedom to choose $q_{\mathscr{W}}$ between 0 and $M-1$, that the only nontrivial solution for even $M$ is $q_{\mathscr{W}}=M / 2$. For odd $M$ there is no non-trivial solution. Since the superpotential charge is given by $q_{\mathscr{W}}=2 q_{\theta}$, the order $M$ has to be divisible by 4 . Hence we can focus on

$$
M=4 \times \text { integer and } q_{\theta}=M / 4
$$

in the rest of our discussion.

\subsubsection{No additional condition from $A_{1}^{R}$}

Subtracting $A_{3}^{R}$ from $A_{1}^{R}$ yields

$$
\frac{3}{5}\left[\frac{1}{2}\left(q_{H_{u}}+q_{H_{d}}\right)-11 q_{\theta}\right]+3 q_{\theta}=0 \bmod \eta .
$$

Since $M$ is even and $q_{H_{u}}+q_{H_{d}}=0 \bmod M$ by (2.3d), this equation is equivalent to

$$
3 k M+2(15-33) q_{\theta}=5 \ell M
$$

with some integers $k$ and $\ell$. That is,

$$
36 q_{\theta}=[3 k-5 \ell] M=\mathbb{Z} \cdot M .
$$

For a given order $M$, this relation constrains $q_{\theta}$. However, we know already from our discussion below Equation (2.14) that $M$ needs to be an integral multiple of 4, such that (2.18) does not lead to an additional constraint. 


\subsection{Family-independent symmetries and proton decay}

Let us now assume further that the discrete symmetry be Abelian, i.e. of $\mathbb{Z}_{M}^{R}$ type, with family-independent charges. Assuming the presence of Yukawa couplings, symmetries with the above properties have automatically the virtue of solving the dimension five proton decay problem of the MSSM, as we will see in the following.

The requirement that up- and down-type Yukawa couplings be allowed,

$$
\begin{aligned}
2 q_{10}+q_{H_{u}} & =q_{\mathscr{W}} \bmod M, \\
q_{10}+q_{\overline{5}}+q_{H_{d}} & =q_{\mathscr{W}} \bmod M,
\end{aligned}
$$

implies

$$
3 q_{10}+q_{\overline{5}}+q_{H_{u}}+q_{H_{d}}=2 q_{\mathscr{W}} \bmod M .
$$

Imposing (2.3d $)$ gives

$$
3 q_{10}+q_{\overline{5}}=2 q_{\mathscr{W}} \quad \bmod M \neq q_{\mathscr{W}} \bmod M,
$$

for an $R$ symmetry (i.e. for $q_{\mathscr{W}} \neq 0 \bmod M$ ), showing that the troublesome dimension five operators $101010 \overline{5}$ are automatically forbidden whenever the Yukawa couplings are allowed. In [8] the same conclusion was obtained from anomaly cancellation.

Recalling further that $2 q_{\mathscr{W}}=4 q_{\theta}=M$ leads us to the conclusion that

$$
q_{\overline{5}}=-3 q_{10} \bmod M .
$$

This means that the contributions of matter fields to the anomaly coefficients (2.5) vanish, and that the universal anomaly coefficients are simply given by

$$
A_{i}^{R}=\rho=q_{\theta} \bmod M / 2
$$

for $1 \leq i \leq 3$.

Next, one can also discuss proton decay originating from the dimension four operator $\mathbf{1 0} \overline{\mathbf{5}} \overline{\mathbf{5}}$. This operator has $R$ charge

$$
q_{10}+2 q_{\overline{5}}=-5 q_{10} \bmod M .
$$

Hence, these operators are also forbidden if $-5 q_{10} \neq q_{\mathscr{W}} \bmod M$, or equivalently $10 q_{10} \neq k M$ with $k$ odd.

\section{$2.4 \quad$ Imposing $\mathrm{SO}(10)$ relations}

Let us now comment on the special case that the $\mathbb{Z}_{M}^{R}$ symmetry commutes with $\mathrm{SO}(10)$ for the matter fields, i.e. $q_{\mathbf{1 0}}=q_{\overline{5}}=q_{\mathbf{1 6}}$. The requirements that the up-type and down-type quark Yukawa couplings be allowed imply that $q_{H_{u}}=q_{H_{d}}=: q_{H}(\bmod M)$. Furthermore, from the anomaly universality condition (2.13b) we find $q_{H}=q_{\mathscr{W}} \bmod \eta$. In the following, we consider two cases: in case (i) we demand in addition the Weinberg neutrino mass operator, and in case (ii) a Giudice-Masiero-like mechanism. 
(i) If we require the Weinberg neutrino mass operator, i.e. $2 q_{16}+2 q_{H}=q_{\mathscr{W}} \bmod M$, we find $M=4 m, m \in \mathbb{N}$ and

$$
q_{\theta}=m, q_{\mathscr{W}}=2 m, q_{H}=0 \text { and } q_{16}=m .
$$

This symmetry automatically allows for the Giudice-Masiero term and the universal anomaly coefficients $A_{i}^{R}=m \neq 0$ indicate a discrete GS mechanism. The simplest case $m=1$ is the $\mathbb{Z}_{4}^{R}$ symmetry discussed in [20, 21]. All other cases are just trivial extensions as long as one considers the MSSM states only. Of course, if additional states are introduced, they can have $\mathbb{Z}_{4 m}^{R}$ charges in such a way that one cannot reduce it to $\mathbb{Z}_{4}^{R}$. Another version of the uniqueness proof of $\mathbb{Z}_{4}^{R}$ can be found in [8]. However, the analysis in [8] assumed that $q_{\theta}=1$. Here we show that uniqueness also survives the generalization to general $q_{\theta} \neq 1$.

(ii) If we do not require the Weinberg neutrino mass operator but a Giudice-Masierolike mechanism, i.e. $2 q_{H}=0 \bmod M$, there are two cases: both cases have $M=4 m$, $m \in \mathbb{N}, q_{\theta}=m$ and $q_{\mathscr{W}}=2 m$. In addition, in the first case we get $q_{H}=0$ as discussed above in case (i), and in the second one we find $q_{H}=M / 2=2 \mathrm{~m}$ and $q_{\mathbf{1 6}}=2 \ell \mathrm{m}$ with $\ell \in \mathbb{Z}$. However, this choice forbids the Weinberg neutrino mass operator.

\subsection{Non-perturbative holomorphic $\mu$ term}

If the above discrete $R$ symmetry appears anomalous, i.e. if anomaly freedom is due to a GS mechanism (see appendix B for a discussion of its discrete variant), then such holomorphic contributions will appear as arising at the non-perturbative level [8, 21]. To see this, recall that the superfield $S$ containing the axion $a$, i.e. $\left.S\right|_{\theta=0}=s+\mathrm{i} a$, needs to enter the gauge-kinetic function, or, in other words, $\mathscr{L} \supset \int \mathrm{d}^{2} \theta f_{S} S W_{\alpha} W^{\alpha}$ (with some coefficient $f_{S}$ ). Non-invariant terms in the superpotential can be made invariant by multiplying them by $\mathrm{e}^{-b S}$ with appropriate $b$. As $s$ controls $1 / g^{2}$ such terms go like $\mathrm{e}^{-b^{\prime} / g^{2}}$, i.e. have the form of instanton contributions. This then fits nicely into the scheme of dynamical supersymmetry breaking [22] (see also the more recent discussion on "retrofitting" [23]), where the scale for supersymmetry breaking is set by a gaugino condensate [24], or a more complicated dynamical term (see e.g. [25] for a review of simple models).

\subsection{Small Dirac neutrino Yukawa couplings}

By relating them to supersymmetry breaking one may explain suppressed neutrino Dirac Yukawa couplings [26 28]. That is, similarly to the $\mu$ term, one can get effective Dirac neutrino Yukawa couplings from the Kähler potential terms

$$
K \supset k_{L H_{u} \bar{\nu}} \frac{X^{\dagger}}{M_{\mathrm{P}}^{2}} L H_{u} \bar{\nu}+\text { h.c. }
$$

as well as

$$
K \supset k_{H_{d}^{\dagger} L \bar{\nu}} \frac{1}{M_{\mathrm{P}}} H_{d}^{\dagger} L \bar{\nu}+\text { h.c. . }
$$


Here, in an obvious notation, $\bar{\nu}$ denotes the right-handed neutrino superfield(s), $k_{L H_{u} \bar{\nu}}$ and $k_{H_{d}^{\dagger} L \bar{\nu}}$ are dimensionless coefficients, and we suppress flavor indices. The first term (2.26a) leads to Dirac neutrino masses when $X$ attains its $F$-term VEV, $\left\langle F_{X}\right\rangle \sim$ $m_{3 / 2} M_{\mathrm{P}}$, while in the case of (2.26b) one has to observe that, due to the presence of the 'non-perturbative' $\mu$ term, also $H_{d}$ attains an $F$ term VEV, $\left\langle F_{H_{d}}\right\rangle \sim \mu\left\langle H_{u}\right\rangle \sim m_{3 / 2} v_{\mathrm{EW}}$. As $q_{H_{u}}+q_{H_{d}}=0 \bmod M$, both terms are allowed if $q_{\bar{\nu}}+q_{H_{u}}+q_{L}=0 \bmod M$, which is precisely the condition that an effective holomorphic $Y_{\nu}$ term is allowed. Altogether we find, analogous to what we have discussed around (2.2), that effective neutrino Yukawa couplings

$$
Y_{\nu} \sim \frac{m_{3 / 2}}{M_{\mathrm{P}}} \sim \frac{\mu}{M_{\mathrm{P}}}
$$

will arise. For $m_{3 / 2}$ in the multi-TeV range this can lead to realistic Dirac neutrino masses. If we are to connect the suppression of $Y_{\nu}$ to the smallness of the $\mu$ term, it is natural to assume that the neutrino Yukawa coupling is forbidden by the same $R$ symmetry that also forbids $\mu$. As discussed above, $L H_{u} \bar{\nu}$ has to have $R$ charge 0 . Moreover, there will also be holomorphic contributions to the Yukawa coupling. That is, even if both $k_{L H_{u} \bar{\nu}}$ and $k_{H_{d}^{\dagger} L \bar{\nu}}$ vanish, Dirac Yukawa couplings of the order $m_{3 / 2} / M_{\mathrm{P}}$ will get induced, where, as in our discussion of the $\mu$ term, $m_{3 / 2}$ represents the order parameter for $R$ symmetry breaking.

\subsection{Discussion}

We have surveyed anomaly-free symmetries which forbid the $\mu$ term and are consistent with the Giudice-Masiero mechanism and $\mathrm{SU}(5)$. We find that these are discrete $R$ symmetries $\mathbb{Z}_{M}^{R}$ with $M=4 m, m \in \mathbb{N}$. The $R$ charges of the $H_{u} H_{d}$ are such that one expects a holomorphic contribution to the $\mu$ term of similar size. That is, the Giudice-Masiero mechanism strongly suggests the presence of additional holomorphic contributions to the effective $\mu$ term!

Assuming further that the symmetries allow the up- and down-type Yukawa couplings and commute with flavor we find that they automatically forbid the troublesome dimension five proton decay operators and in many cases those of dimension four. Interestingly, all these symmetries require a GS axion for anomaly cancellation. That is, these symmetries appear to be broken at the non-perturbative level. In other words, imposing compatibility with the Giudice-Masiero mechanism leads us to a situation in which a holomorphic $\mu$ term appears at the non-perturbative level, i.e. in a way the Giudice-Masiero term is unnecessary.

\section{Classification and models}

In this section, we explore anomaly-free discrete symmetries that solve some of the most severe problems of the MSSM. We will demand that the symmetry

1. is flavor-universal and Abelian, i.e. a $\mathbb{Z}_{M}^{R}$ symmetry; 
2. commutes with $\mathrm{SU}(5)$;

3. forbids the $\mu$ term perturbatively;

4. allows the usual Yukawa couplings;

After revisiting in Section 3.1 the scan performed in [8], where Majorana neutrinos were considered, we turn to the Dirac case in Section 3.2 .

\subsection{Models with Majorana neutrinos}

In [8, 21], anomaly-free discrete $R$ symmetries with $q_{\theta}=1$ were studied which satisfy the requirements 14 and in addition

5. allow the Weinberg neutrino mass operator.

It was found that there are only five phenomenologically attractive symmetries that commute with SU(5), one of which, a simple $\mathbb{Z}_{4}^{R}$ symmetry, commutes also with $\mathrm{SO}(10)$. Further, the $\mu$ term, while perturbatively forbidden, appears at the non-perturbative level in four out of the five symmetries, and thus can explain its suppression. There is one symmetry which is anomaly-free without GS contribution; here anomaly freedom requires the number of generations to be a multiple of 3 [29] (for a similar connection between the number of generations and anomaly-free non- $R$ symmetries see [6, 30]).

In the classification of $\left[8, \mathbb{Z}_{4}^{R}\right.$ appears to be particularly attractive. Apart from the fact that it is the unique solution that commutes with $\mathrm{SO}(10)$, only $\mathbb{Z}_{4}^{R}$ provides a real solution to the $\mu$ problem. In this case, the discrete charges of $H_{u}$ and $H_{d}$ add up to $0 \bmod M=4$ such that the $\mu$ parameter will be of the order of the gravitino mass, i.e. the order parameter of $R$ breaking. This feature is not shared by the other four $\mathbb{Z}_{M}^{R}$ symmetries, as also can be seen from our analysis in Section 2.4. In particular, it was argued that $\mu \sim m_{3 / 2}$ for the case of $\mathbb{Z}_{4}^{R}$. To substantiate these claims, an explicit string model with exact MSSM spectrum and the $\mathbb{Z}_{4}^{R}$ symmetry was constructed in which the relation $\mu \sim\langle\mathscr{W}\rangle \sim m_{3 / 2}$ is due to gauge invariance in extra dimensions [31.

Assuming in addition a Giudice-Masiero-like mechanism, one can see that $\mathbb{Z}_{4}^{R}$ is the unique solution also for general $q_{\theta}$ as follows. From the requirement that the Weinberg operator be allowed we infer that

$$
2 q_{\overline{5}}+2 q_{H_{u}}=2 q_{\theta} \quad \bmod M \curvearrowright q_{\overline{5}}=q_{\theta}-q_{H_{u}} \quad \bmod M / 2 .
$$

On the other hand, from the down-type Yukawa coupling it follows

$$
q_{10}=-q_{\overline{5}}-q_{H_{d}}+2 q_{\theta} \quad \bmod M \stackrel{\text { 3.1 }}{=} q_{\theta}+q_{H_{u}}-q_{H_{d}} \bmod M / 2 .
$$

Demanding that the up-type Yukawa coupling be allowed leads to

$$
\begin{aligned}
q_{H_{u}} & =2 q_{\theta}-2 q_{10} \quad \bmod M \\
& \stackrel{3.2}{=}-2 q_{H_{u}}+2 q_{H_{d}} \quad \bmod M=-4 q_{H_{u}} \quad \bmod M,
\end{aligned}
$$


such that $5 q_{H_{u}}=0 \bmod M$. This means that $q_{H_{u}}=0 \bmod M$ unless the order is a multiple of 5 . In the latter case we can write the $\mathbb{Z}_{M}^{R}$ symmetry as $\mathbb{Z}_{5} \times \mathbb{Z}_{M / 5}^{R}$ where the $\mathbb{Z}_{5}$ factor is a non- $R$ symmetry. Hence we can focus on $q_{H_{u}}=0 \bmod M$, which implies, by (2.3d), that $q_{H_{d}}=0 \bmod M$. Then Equations (3.1) and (3.2) imply

$$
q_{10}=q_{\overline{5}}=q_{\theta} \bmod M .
$$

That is, the symmetry commutes with $\mathrm{SO}(10)$ in the matter sector. We already know from our discussion in Section 2.4 that the only meaningful $R$ symmetry with this property is $\mathbb{Z}_{4}^{R}$.

We also scanned the discrete $\mathbb{Z}_{M}^{R}$ symmetries up to order 200 with general $q_{\theta}$ without assuming a Giudice-Masiero-like mechanism. We obtain, apart from the symmetries of Tables 2.1 and 2.2 of [8], only a few new symmetries. However, as we show in the following in an example, these additional symmetries are redundant: consider a $\mathbb{Z}_{20}^{R}$ symmetry with $\left(q_{\mathbf{1 0}}, q_{\overline{\mathbf{5}}}, q_{H_{u}}, q_{H_{d}}, q_{\theta}\right)=(1,17,8,52,5)$. This is equivalent to a $\mathbb{Z}_{4}^{R} \times \mathbb{Z}_{5}$ symmetry with charge assignment $((1,3),(1,1),(0,4),(0,1),(1,0))$. The $\mathbb{Z}_{5}$ is nothing but the non-trivial center of SU(5), i.e. it does not forbid any couplings (see the discussion in [16, 32]) and the (non-trivial) $\mathbb{Z}_{4}^{R}$ factor is the one just discussed in the last paragraph.

\subsection{Models with Dirac neutrinos}

By modifying the above conditions, i.e. by demanding that the symmetry

5. forbids the Weinberg neutrino mass operator perturbatively

and

6. is compatible with the Giudice-Masiero mechanism

we obtain further interesting discrete $R$ symmetries. Some sample symmetries are listed in Table 1. Anomaly-free (non- $R$ ) $\mathbb{Z}_{N}$ symmetries which allow for Dirac neutrino Yukawa couplings have been discussed in [33]. The symmetries of Table 1 are inequivalent. One way of verifying this is to check whether or not two given charge assignments are equivalent by computing their Hilbert superpotential basis [34. Only if the bases coincide, the assignments are equivalent. In the case of $R$ symmetries, the Hilbert superpotential basis comprises homogeneous and inhomogeneous elements, or monomials. Every possible superpotential term contains precisely one inhomogeneous monomial and an arbitrary number of homogeneous monomials. In appendix C we list the Hilbert superpotential basis for examples with the $\mathbb{Z}_{12}^{R}$ symmetries.

\subsubsection{Comments on the $\mathbb{Z}_{8}^{R}$ symmetry}

One of simplest charge assignments appears to be the one of the $\mathbb{Z}_{8}^{R}$ symmetry. Clearly the usual Yukawa couplings $\mathbf{1 0 1 0} H_{u}$ and $\mathbf{1 0} \overline{\mathbf{5}} H_{d}$ are allowed. Further, the Higgs bilinear $H_{u} H_{d}$ has $R$ charge $0 \bmod 8$. If we assign the right-handed neutrino $\bar{\nu} R$ 
(a) $\mathbb{Z}_{M}^{R}$ symmetries.

\begin{tabular}{cccccccc}
$M$ & $q_{\mathbf{1 0}}$ & $q_{\overline{\mathbf{5}}}$ & $q_{H_{u}}$ & $q_{H_{\boldsymbol{d}}}$ & $q_{\theta}$ & $\rho$ & $q_{\bar{\nu}}$ \\
\hline 4 & 0 & 0 & 2 & 2 & 1 & 1 & 2 \\
4 & 2 & 2 & 2 & 2 & 1 & 1 & 0 \\
8 & 1 & 5 & 2 & 6 & 2 & 2 & 1 \\
12 & 1 & 9 & 4 & 8 & 3 & 3 & 11 \\
12 & 2 & 6 & 2 & 10 & 3 & 3 & 4 \\
12 & 4 & 0 & 10 & 2 & 3 & 3 & 2 \\
16 & 1 & 13 & 6 & 10 & 4 & 4 & 13 \\
24 & 1 & 21 & 10 & 14 & 6 & 6 & 17 \\
28 & 1 & 25 & 12 & 16 & 7 & 7 & 19 \\
28 & 2 & 22 & 10 & 18 & 7 & 7 & 24 \\
28 & 4 & 16 & 6 & 22 & 7 & 7 & 6 \\
32 & 1 & 29 & 14 & 18 & 8 & 8 & 21 \\
36 & 1 & 33 & 16 & 20 & 9 & 9 & 23 \\
36 & 2 & 30 & 14 & 22 & 9 & 9 & 28 \\
36 & 4 & 24 & 10 & 26 & 9 & 9 & 2
\end{tabular}

(b) Residual symmetries.

\begin{tabular}{cccccc}
$M^{\prime}$ & $q_{\mathbf{1 0}}$ & $q_{\overline{\mathbf{5}}}$ & $q_{H_{u}}$ & $q_{H_{d}}$ & $q_{\bar{\nu}}$ \\
\hline 2 & 0 & 0 & 0 & 0 & 0 \\
2 & 0 & 0 & 0 & 0 & 0 \\
4 & 1 & 1 & 2 & 2 & 1 \\
6 & 1 & 3 & 4 & 2 & 5 \\
3 & 1 & 0 & 1 & 2 & 2 \\
3 & 2 & 0 & 2 & 1 & 1 \\
8 & 1 & 5 & 6 & 2 & 5 \\
12 & 1 & 9 & 10 & 2 & 5 \\
14 & 1 & 11 & 12 & 2 & 5 \\
7 & 1 & 4 & 5 & 2 & 5 \\
7 & 2 & 1 & 3 & 4 & 3 \\
16 & 1 & 13 & 14 & 2 & 5 \\
18 & 1 & 15 & 16 & 2 & 5 \\
9 & 1 & 6 & 7 & 2 & 5 \\
9 & 2 & 3 & 5 & 4 & 1
\end{tabular}

Table 1: Classification of anomaly-free discrete $R$ symmetries that forbid neutrino masses perturbatively. We restrict to orders $\leq 36$. (a) shows some sample symmetries. The equality between $q_{\theta}$ and $\rho$ is due to Equation (2.23). The charge of the right-handed neutrino superfield $\bar{\nu}$ is determined by the requirement that $q_{\bar{\nu}}+q_{H_{u}}+q_{L}=0 \bmod M$ (cf. the discussion below (2.26) ). In (b) we display the residual symmetries that remain after the ('hidden sector') superpotential acquires its VEV.

charge 1, the Dirac neutrino Yukawa coupling will also be induced by $R$ breaking. That is, we will have an effective superpotential which is schematically of the form

$$
\mathscr{W}_{\text {eff }} \sim m_{3 / 2} H_{u} H_{d}+\frac{m_{3 / 2}}{M_{\mathrm{P}}} L H_{u} \bar{\nu}+\frac{m_{3 / 2}}{M_{\mathrm{P}}^{2}} Q Q Q L .
$$

Here we suppress flavor indices. Once the superpotential of the hidden sector acquires a $\mathrm{VEV}$, the $\mathbb{Z}_{8}^{R}$ is spontaneously broken down to a $\mathbb{Z}_{4}$ symmetry under which all matter fields have charge 1 and the Higgs fields have charge 2 (Table 1 (b)). Of course, this symmetry gets broken down to the usual matter (or ' $R$ ') parity once the Higgs scalars attain their VEVs.

The Hilbert superpotential basis [34] for this model (setting all quarks to zero) is given by the inhomogeneous monomials

$$
\bar{\nu}^{4} ;(L L \bar{E}) \bar{\nu} ;\left(L H_{d} \bar{E}\right) ;(L L \bar{E})^{4} ;(L L \bar{E})^{2}\left(L H_{u}\right)^{2} ;\left(L H_{u}\right)^{4}
$$


while the homogeneous monomials are

$$
\begin{aligned}
& \bar{\nu}^{8} ;\left(L H_{u}\right) \bar{\nu} ;\left(L H_{u}\right)^{8} ;(L L \bar{E})^{5} \bar{\nu} ;(L L \bar{E})^{4}\left(L H_{d} \bar{E}\right) ; \\
& H_{u} H_{d} ;(L L \bar{E}) \bar{\nu}^{5} ;\left(L H_{d} \bar{E}\right) \bar{\nu}^{4} ;(L L \bar{E})^{2}\left(L H_{d} \bar{E}\right)\left(L H_{u}\right)^{2} ; \\
& \quad(L L \bar{E})^{8} ;\left(L H_{d} \bar{E}\right)^{2} ;(L L \bar{E})\left(L H_{d} \bar{E}\right) \bar{\nu} ;(L L \bar{E})^{2} \bar{\nu}^{2} ; \\
& (L L \bar{E})^{3}\left(L H_{u}\right) ;\left(L H_{d} \bar{E}\right)\left(L H_{u}\right)^{4} ;(L L \bar{E})\left(L H_{u}\right)^{3} .
\end{aligned}
$$

Furthermore, there will be Kähler potential terms

$$
K \supset X^{\dagger}\left(\frac{k_{H_{u} H_{d}}}{M_{\mathrm{P}}} H_{u} H_{d}+\frac{k_{L H_{u} \bar{\nu}}}{M_{\mathrm{P}}^{2}} L H_{u} \bar{\nu}+\frac{k_{Q Q Q L}}{M_{\mathrm{P}}^{3}} Q Q Q L\right)+\text { h.c. }
$$

with $X$ denoting the field that breaks supersymmetry, $k_{H_{u} H_{d}}, k_{L H_{u} \bar{\nu}}$ and $k_{Q Q Q L}$ being coefficients (and the flavor indices again are suppressed). The $k_{H_{u} H_{d}}$ term is nothing but the famous Giudice-Masiero term [7].

An important feature of this setting is that lepton number is violated at the quartic level, but bilinear lepton number violating terms are absent. That is, this model predicts the absence of neutrinoless double $\beta$ decays. On the other hand, lepton number is not a good symmetry, which might have, for instance, important implications for the early universe.

Let us also note that the coefficients in the above Kähler potential are not necessarily of order unity. In specific string constructions, these coefficients can in fact be as large as $\mathcal{O}(10-100)$ due to the presence of copious heavy states and/or combinatorical factors (cf. the discussion in [35]), enabling realistic predictions for neutrino masses in the sub-eV range.

\subsubsection{Comments on the $\mathbb{Z}_{4}^{R}$ symmetries}

Both $\mathbb{Z}_{4}^{R}$ symmetries of Table 1 are problematic as they allow some $R$ parity violating couplings. In particular, the first $\mathbb{Z}_{4}^{R}$ allows for bi-linear $R$ parity violation, i.e. the $\overline{\mathbf{5}} H_{u}$ coupling, while the second $\mathbb{Z}_{4}^{R}$ admits the tri-linear $R$ parity violating terms $\mathbf{1 0} \overline{\mathbf{5}} \overline{\mathbf{5}}$. In addition, both settings allow for a non-perturbative neutrino bilinear $\bar{\nu} \bar{\nu}$. That is, these symmetries can give us a non-perturbative Majorana neutrino mass term, which might be relevant for the construction of models realizing a TeV-scale see-saw scenario. Given our previous discussion, a straightforward possibility of rectifying this is to amend the settings by the residual $\mathbb{Z}_{4}$ symmetry from above (Table 21).

The $\mathbb{Z}_{4}^{R}$ symmetries originally give us two inequivalent Hilbert superpotential bases, however, amending the settings by the above-mentioned $\mathbb{Z}_{4}$ symmetry leads to the same basis. Therefore, both $\mathbb{Z}_{4}^{R} \times \mathbb{Z}_{4}$ symmetries give us the inhomogeneous monomials

$$
\left(L H_{d} \bar{E}\right) ;(L L \bar{E}) \bar{\nu} ;(L L \bar{E})\left(L H_{u}\right)^{3} ;(L L \bar{E})^{3}\left(L H_{u}\right) \text {, }
$$


(a) First $\mathbb{Z}_{4}^{R}$.

\begin{tabular}{cccccccc} 
& $q_{\mathbf{1 0}}$ & $q_{\overline{\mathbf{5}}}$ & $q_{H_{u}}$ & $q_{H_{d}}$ & $q_{\theta}$ & $\rho$ & $q_{\bar{\nu}}$ \\
\hline $\mathbb{Z}_{4}^{R}$ & 0 & 0 & 2 & 2 & 1 & 1 & 2 \\
$\mathbb{Z}_{4}$ & 1 & 1 & 2 & 2 & 0 & 0 & 1
\end{tabular}

(b) Second $\mathbb{Z}_{4}^{R}$.

\begin{tabular}{cccccccc} 
& $q_{\mathbf{1 0}}$ & $q_{\overline{\mathbf{5}}}$ & $q_{H_{u}}$ & $q_{H_{d}}$ & $q_{\theta}$ & $\rho$ & $q_{\bar{\nu}}$ \\
\hline $\mathbb{Z}_{4}^{R}$ & 2 & 2 & 2 & 2 & 1 & 1 & 0 \\
$\mathbb{Z}_{4}$ & 1 & 1 & 2 & 2 & 0 & 0 & 1
\end{tabular}

Table 2: $\mathbb{Z}_{4}$ extensions of the $\mathbb{Z}_{4}^{R}$ symmetries of Table 1 ,

whereas the homogeneous ones are given by

$$
\begin{aligned}
& \bar{\nu}^{4} ; H_{u} H_{d} ;\left(L H_{u}\right) \bar{\nu} ;\left(L H_{u}\right)^{4} ;\left(L H_{d} \bar{E}\right)\left(L H_{u}\right)(L L \bar{E})^{3} ; \\
& \left(L H_{d} \bar{E}\right)^{2} ;(L L \bar{E})\left(L H_{d} \bar{E}\right) \bar{\nu} ;(L L \bar{E})^{2} \bar{\nu}^{2} ; \\
& (L L \bar{E})^{2}\left(L H_{u}\right)^{2} ;\left(L H_{d} \bar{E}\right)\left(L H_{u}\right)^{3}(L L \bar{E}) ;(L L \bar{E})^{4} .
\end{aligned}
$$

As before in our $\mathbb{Z}_{8}^{R}$ setting, bilinear lepton number violating terms are absent. In both cases this feature is due to the (anomaly-free non- $R$ ) $\mathbb{Z}_{4}$ symmetry, which commutes with $\mathrm{SO}(10)$ for the matter fields and is a consistent symmetry of the MSSM. Unlike the $R$ symmetries, this symmetry does not forbid the $\mu$ term nor the dimension five proton decay operators.

\section{Summary}

The MSSM provides a very attractive scheme for physics beyond the standard model. However, in order to address its shortcomings, one, arguably, has to impose additional symmetries. Motivated by the structure of matter and the attractive picture of gauge unification, we have considered symmetries that commute with $\mathrm{SU}(5)$ in the matter sector. From the requirement of anomaly freedom it follows that only discrete $R$ symmetries can forbid the $\mu$ term. We also pointed out that anomaly matching for $R$ symmetries in $\mathrm{SU}(5)$ symmetric models implies the existence of split multiplets below the GUT scale, with the simplest option being that a pair of Higgs doublets cancels the anomaly mismatch between the gauginos. Further demanding that a $\mu$ term of the order of the gravitino mass arises from supersymmetry breaking, i.e. either from the Kähler potential or from the non-trivial superpotential VEV in the 'hidden sector', we showed that the Higgs bilinear $H_{u} H_{d}$ has to carry trivial $R$ charge. We find that discrete $R$ symmetries with these properties automatically forbid dimension-five proton decay operators once the usual Yukawa couplings are allowed. Even more, all symmetries appear anomalous such that a holomorphic $\mu$ term gets induced at the non-perturbative level. That is, demanding compatibility with the Giudice-Masiero mechanism brings us to the situation in which a $\mu$ term of the desired magnitude appears even without the Giudice-Masiero term in the Kähler potential.

We then discussed neutrino masses in the emerging MSSM models amended by discrete $R$ symmetries. Restricting ourselves to flavor-universal Abelian, i.e. $\mathbb{Z}_{M}^{R}$, sym- 
metries we find that, by demanding that the Weinberg operator $L H_{u} L H_{u}$ be allowed, there exists only one possible symmetry, namely a $\mathbb{Z}_{4}^{R}$ symmetry. Following a different approach, this $\mathbb{Z}_{4}^{R}$ has also recently been shown to be the unique anomaly-free symmetry that commutes with $\mathrm{SO}(10)$ [21]. The proof in [21] assumed that the charge of the superspace coordinate $\theta$ can always be set 1 , which we find to be too strong a requirement. However, we find that, if one is to allow for arbitrary $\theta$ charges, this only leads to trivial extensions of $\mathbb{Z}_{4}^{R}$, such that the uniqueness of $\mathbb{Z}_{4}^{R}$ still prevails.

If one requires instead the discrete symmetry to forbid the Weinberg operator, one can explain small Dirac neutrino masses. In particular, we successfully obtain a relation between the smallness of Dirac neutrino Yukawa couplings and the $\mu$ term which is based on anomaly-free discrete $R$ symmetries with the above properties. Specifically, we find a class of anomaly-free discrete symmetries in which the appealing relations $\mu \sim\langle\mathscr{W}\rangle / M_{\mathrm{P}}^{2} \sim m_{3 / 2}$ and $Y_{\nu} \sim \mu / M_{\mathrm{P}}$ naturally emerge.

\section{Acknowledgments}

We would like to thank Maximilian Fallbacher and Hans Peter Nilles for useful discussions. M.R. would like to thank the UC Irvine, where part of this work was done, for hospitality. M.-C.C. would like to thank TU München, where part of the work was done, for hospitality. This work was partially supported by the DFG cluster of excellence "Origin and Structure of the Universe" and the Graduiertenkolleg "Particle Physics at the Energy Frontier of New Phenomena" by Deutsche Forschungsgemeinschaft (DFG). P.V. is supported by SFB grant 676. The work of M.-C.C. was supported, in part, by the U.S. National Science Foundation under Grant No. PHY-0970173. M.-C.C., M.R. and P.V. would like to thank the Aspen Center for Physics for hospitality and support. M.-.C.C. thanks the Galileo Galilei Institute for Theoretical Physics for the hospitality. This research was done in the context of the ERC Advanced Grant project "FLAVOUR" (267104).

\section{A Anomaly coefficients for $\mathbb{Z}_{M}^{R}$ symmetries with ar- bitrary $q_{\theta}$}

The anomaly conditions for discrete $R$ symmetries depend on $q_{\theta}$. Consider a $\mathbb{Z}_{M}^{R}$ symmetry, under which the superpotential transforms as

$$
\mathscr{W} \rightarrow \mathrm{e}^{2 \pi \mathrm{i} q_{\mathscr{W}} / M} \mathscr{W}
$$

with $q_{\mathscr{W}}=2 q_{\theta}$ (such that $\int \mathrm{d}^{2} \theta \mathscr{W}$ is invariant). Superfields $\Phi^{(f)}=\phi^{(f)}+\sqrt{2} \theta \psi^{(f)}+$ $\theta \theta F^{(f)}$ transform as

$$
\Phi^{(f)} \rightarrow \mathrm{e}^{2 \pi \mathrm{i} q^{(f)} / M} \Phi^{(f)} .
$$

Correspondingly, the fermions transform as

$$
\psi^{(f)}=\mathrm{e}^{2 \pi \mathrm{i}\left(q^{(f)}-q_{\theta}\right) / M} \psi^{(f)} .
$$


The anomaly coefficients hence read (cf. [8, Appendix B], where the anomaly coefficients for the special case $q_{\theta}=1$ are shown)

$$
\begin{aligned}
A_{G-G-\mathbb{Z}_{M}^{R}} & =\sum_{f} \ell\left(\boldsymbol{r}^{(f)}\right) \cdot\left(q^{(f)}-q_{\theta}\right)+q_{\theta} \ell(\operatorname{adj} G) \\
A_{\mathrm{U}(1)-\mathrm{U}(1)-\mathbb{Z}_{M}^{R}} & =\sum_{f}\left(Q^{(f)}\right)^{2} \operatorname{dim}\left(\boldsymbol{r}^{(f)}\right) \cdot\left(q^{(f)}-q_{\theta}\right) \\
A_{\text {grav }-\operatorname{grav}-\mathbb{Z}_{M}^{R}} & =-21 q_{\theta}+q_{\theta} \sum_{G} \operatorname{dim}(\operatorname{adj} G)+\sum_{f} \operatorname{dim}\left(\boldsymbol{r}^{(f)}\right) \cdot\left(q^{(f)}-q_{\theta}\right) .
\end{aligned}
$$

Here $q^{(f)}$ denote the $\mathbb{Z}_{M}^{R}$ charges of the superfields, the charges of the corresponding fermions are shifted by $q_{\theta}, q_{\psi^{(f)}}=q^{(f)}-q_{\theta}$. In Equation (A.4a), $\ell\left(\boldsymbol{r}^{(f)}\right)$ denotes the Dynkin index of representation $\boldsymbol{r}^{(f)}$ normalized to $\ell(\boldsymbol{N})=\frac{1}{2}$ for the fundamental representation $\boldsymbol{N}$ of $\mathrm{SU}(N)$ and $\ell(\operatorname{adj} G)=c_{2}(G)$ represents the contribution from the gauginos, i.e. $\ell(\operatorname{adj} \mathrm{SU}(N))=N$. The first and second terms on the right-hand side of Equation (A.4C) represent the contributions from the gravitino and gauginos.

\section{B Green-Schwarz anomaly cancellation and anomaly universality}

In this Appendix, we discuss the discrete Green-Schwarz (GS) anomaly cancellation mechanism, following [8]. We start by reviewing the GS mechanism for a continuous $\mathrm{U}(1)$ symmetry in B.1. In B.2 we discuss the discrete version while B.3 is dedicated to the discussion of anomaly universality.

\section{B.1 Anomaly cancellation for 'anomalous U(1)' symmetries}

We start by discussing the mixed anomaly coefficients $G-G-\mathrm{U}(1)_{\text {anom }}$ for a simple gauge group $G$. There will be an axion $a$ which couples to the field strength of $G$ via

$$
\mathscr{L}_{\text {axion }} \supset \frac{a}{8} F^{b} \widetilde{F}^{b}
$$

A possible prefactor can be absorbed in the normalization of $a$, which we do not specify here. Consider now the gauge transformation

$$
\psi^{(f)} \rightarrow \mathrm{e}^{\mathrm{i} \alpha(x) Q_{\text {anom }}^{(f)}} \psi(f),
$$

where $\psi^{(f)}(1 \leq f \leq F)$ denotes the fermions of the theory and $Q_{\text {anom }}^{(f)}$ their charges. The crucial property of the axion $a$ is that it shifts under (B.2) as

$$
a \rightarrow a+\frac{1}{2} \delta_{\mathrm{GS}} \alpha(x)
$$


We can now fix the the Green-Schwarz coefficient $\delta_{\mathrm{GS}}$ from the requirement of invariance of the full quantum theory. It follows from (B.3) that, under a U(1)anom transformation with parameter $\alpha$, the axionic Lagrangean shifts by

$$
\Delta \mathscr{L}_{\text {axion }}=-\frac{\alpha}{16} \delta_{\mathrm{GS}} F^{b} \widetilde{F}^{b} .
$$

The Green-Schwarz term $\delta_{\mathrm{GS}}$ can now be inferred by demanding that the transformation of the axion $a$ cancels the anomalous variation of the path integral measure [36, 37]. The latter can be absorbed in a change of the Lagrangean

$$
\Delta \mathscr{L}_{\text {anomaly }}=\frac{\alpha}{32 \pi^{2}} F^{b} \widetilde{F}^{b} A_{G-G-\mathrm{U}(1)_{\text {anom }}} .
$$

The coefficient $A$ is the anomaly coefficient, given by

$$
A_{G-G-\mathrm{U}(1)_{\mathrm{anom}}}=\sum_{r^{(f)}} \ell\left(\boldsymbol{r}^{(f)}\right) Q_{\mathrm{anom}}^{(f)}
$$

where the sum runs over all irreducible (fermionic) representations $\boldsymbol{r}^{(f)}$ of $G, \ell\left(\boldsymbol{r}^{(f)}\right)$ denotes the Dynkin index of $\boldsymbol{r}^{(f)}$ and $Q_{\text {anom }}^{(f)}$ is the $\mathrm{U}(1)$ anom charge.

The axion shift allows us to cancel the $G-G-\mathrm{U}(1)_{\text {anom }}$ anomaly by demanding $\Delta \mathscr{L}_{\text {anomaly }}+\Delta \mathscr{L}_{\text {axion }}=0$. This fixes the Green-Schwarz constant to be

$$
2 \pi^{2} \delta_{\mathrm{GS}}=A_{G-G-\mathrm{U}(1)_{\mathrm{anom}}} .
$$

\section{B.2 Discrete Green-Schwarz mechanism}

The Green-Schwarz mechanism also works if we replace $\mathrm{U}(1)_{\text {anom }}$ by a discrete $\mathbb{Z}_{M}$. In this case the parameter $\alpha$ is no longer continuous but $\alpha=\frac{2 \pi n}{M}$ with some integer $n$. Of course, there is no gauge field associated with the $\mathbb{Z}_{M}$. The discussion then goes as in the previous subsection. The discrete Green-Schwarz constant is now defined in such a way that under the $\mathbb{Z}_{M}$ transformation of fermions

$$
\psi^{(f)} \rightarrow \mathrm{e}^{-\mathrm{i} \frac{2 \pi}{M} q^{(f)}} \psi^{(f)}
$$

the axion shifts according to

$$
a \rightarrow a+\frac{1}{2} \Delta_{\mathrm{GS}}
$$

where $\Delta_{\mathrm{GS}}$ is fixed only modulo $\eta$,

$$
\pi M \Delta_{\mathrm{GS}} \equiv A_{G-G-\mathbb{Z}_{M}} \bmod \eta
$$

The anomaly coefficients can be obtained from Equation (B.6) by replacing the U(1) anom charges $Q_{\text {anom }}^{(m)}$ by the $\mathbb{Z}_{M}$ charges $q^{(m)}$. 


\section{B.3 Multiple gauge groups and "anomaly universality"}

Let us now discuss the case of multiple gauge groups $G_{i}$. In heterotic string models very often the $\mathrm{U}(1)_{\text {anom }}, \mathbb{Z}_{N}$ or $\mathbb{Z}_{M}^{R}$ anomaly coefficients fulfill certain universality relations,

$$
A_{G_{i}-G_{i}-H}=\rho
$$

for all $i$ and in this section $H$ denotes either $\mathrm{U}(1)_{\text {anom }}, \mathbb{Z}_{N}$ or $\mathbb{Z}_{M}^{R}$. We will refer to (B.11) as "anomaly universality". In a recent paper [38] it has been pointed out correctly that this may not necessarily be the case in general. That is, the anomaly universality (B.11) is not a direct consequence of GS anomaly cancellation.

In detail, multiple gauge groups $G_{i}$ in general do allow us to introduce different couplings $c_{i}$ of the axion $a$ to the various field strengths,

$$
\mathscr{L}_{\text {axion }} \supset \sum_{i} c_{i} \frac{a}{8} F_{i}^{b} \widetilde{F}_{i}^{b}
$$

The requirement that under an $H$ transformation the contribution from the path integral measure gets cancelled by the discrete shift of the axion then implies that

$$
2 \pi^{2} c_{i} \delta_{\mathrm{GS}}=A_{G_{i}-G_{i}-H}
$$

for all $i$. That means that the $c_{i}$ coefficients can be chosen in such a way that the transformation of the path integral measure gets cancelled for each $G_{i}$ gauge factor separately. In particular, one finds (in agreement with [38]) that in general the mixed $A_{G_{i}-G_{i}-H}$ do not need to be universal.

However, if this was the case in a given model, one would spoil the beautiful picture of MSSM gauge coupling unification. Let us spell out the argument in some more detail. In supersymmetry, the Lagrangean (B.12) implies that there are couplings between the superfield $S$ which contains the axion, $\left.S\right|_{\theta=0}=s+\mathrm{i} a$, and the supersymmetric field strengths $W^{(i)}$ associated to the gauge group factors $G_{i}$, i.e.

$$
\mathscr{L}_{\text {axion }} \supset \sum_{i} \int \mathrm{d}^{2} \theta \frac{c_{i}}{8} S W_{\alpha}^{(i)} W^{(i) \alpha} .
$$

Once the real part of $S$ acquires a VEV this will give rise to a non-universal change of the gauge couplings unless the $c_{i}$ coefficients are all equal for the SM gauge group factors $G_{i}=\mathrm{SU}(3)_{\mathrm{C}}, \mathrm{SU}(2)_{\mathrm{L}}$ and $\mathrm{U}(1)_{Y}$. That is, anomaly universality is also required in order not to spoil the beautiful picture of MSSM gauge coupling unification.

Furthermore, there might be model-dependent reasons why the $A_{G_{i}-G_{i}-H}$ can be universal, for instance if all $G_{i}$ come from a (for instance grand unified) simple gauge group, as we assume in the main body of this paper. Then the term

$$
\mathscr{L}_{\text {axion }} \supset a F_{\text {GUT }}^{b} \widetilde{F}_{\text {GUT }}^{b}
$$

is obviously gauge invariant. Hence, the anomalies need to be universal at least at the GUT level, as discussed around Equation (2.10). 
How could this universality possibly be broken? One may now worry about additional terms of the form

$$
\mathscr{L}_{\text {axion }} \supset a\left(\frac{\Phi_{\mathrm{GUT}}}{M} F_{\mathrm{GUT}} \widetilde{F}_{\mathrm{GUT}}\right)
$$

where the operator $\Phi_{\text {GUT }}$ furnishes a non-trivial GUT representation (such as a $\mathbf{2 4 - p l e t}$ of $\mathrm{SU}(5))$ and the parentheses denote a non-trivial contraction of the group indices 3 However, at the GUT level, i.e. for a trivial $\Phi_{\mathrm{GUT}} \mathrm{VEV}$, such a term can not cancel the transformation of the path integral measure by a shift transformation of the axion $a$. In other words, it is not allowed by the symmetries of the action if we require that $a$ shifts. Hence, these operators can not break anomaly universality.

However, there is a second possibility. In higher-dimensional, e.g. in orbifold GUT type, models there can be localized terms which do not respect the GUT symmetry. That is, in settings where the GUT symmetry is broken locally in some regions of compact space such as orbifold fixed points, anomaly non-universality can arise. After integrating over compact space in order to derive the four-dimensional effective action one can indeed arrive at non-universal couplings $c_{i}$ of the axion to the three $F \widetilde{F}$ terms of the standard model. Still, as discussed before, if one is not to spoil the beautiful picture of MSSM gauge coupling unification, the $A_{G_{i}-G_{i}-H}$ coefficients need to be universal and these localized contributions have to be avoided. One possibility to avoid them is "non-local GUT breaking" in extra dimensions, which has been argued to yield the most appealing scenarios of precision gauge unification [40 42. In such scenarios, the localized dangerous GUT-breaking operators do not exist and hence the anomalies are universal 4

Let us also comment on another statement in [38. First, we would like to point out that the number of axions is not related to anomaly universality. Specifically, in the presence of multiple axions, which are available in heterotic compactifications [43,44, one would have to define how they transform under a $\mathrm{U}(1)_{\text {anom }}$ (or discrete) transformation. Since there is only one such transformation, this allows us to identify one unique linear combination of axions, called $a$ as in our discussion above, which shifts while the other 'would-be axions' stay inert. Therefore, the number of axions is not related to the question of anomaly (non-) universality.

Furthermore, the authors of [38] argue that the anomalies cannot be universal both before and after doublet-triplet splitting. We disagree with this statement. First of all, 'before doublet-triplet splitting', i.e. before GUT breaking, there are more states

\footnotetext{
${ }^{3}$ The relative coefficients $c_{i}$ of the axion coupling to the three $F_{i} \widetilde{F}_{i}$ terms of the standard model originating from $\Phi_{\mathrm{GUT}}$ can be inferred from [39].

${ }^{4}$ In Abelian orbifold models such operators can only stem from localized fluxes, which are Abelian (i.e. U(1)) fluxes. Hence, the $A_{G_{i}-G_{i}-H}$ coefficients coincide for all non-Abelian factors $G_{i}$ from each $\mathrm{E}_{8}$ in such models. This is also in agreement with 38 where it is found that, in compactifications of the heterotic $\mathrm{E}_{8} \times \mathrm{E}_{8}$ string on blown-up orbifolds with Abelian fluxes, non-Abelian anomalies of each $\mathrm{E}_{8}$ factor are still universal. Since the relevant assumption in Section 2.2 needed to prove the uniqueness of $\mathbb{Z}_{4}^{R}$ (see also Section 2.2.6) is that $A_{\mathrm{SU}(2)_{\mathrm{L}}-\mathrm{SU}(2)_{\mathrm{L}}-\mathbb{Z}_{M}^{R}}$ and $A_{\mathrm{SU}(3)_{\mathrm{C}}-\mathrm{SU}(3)_{\mathrm{C}}-\mathbb{Z}_{M}^{R}}$ coincide, the uniqueness of $\mathbb{Z}_{4}^{R}$ is also given in such constructions.
} 
around which contribute to the anomalies and anomaly universality follows from gauge invariance under the GUT group, see Equation (B.15). Moreover, in the absence of localized GUT breaking terms, if the anomaly coefficients are universal at the GUT level, where the contributions of extra states have to be taken into account, they should also be so in the MSSM. This is, again, nothing but 't Hooft anomaly matching (see Section 2.2.3). In fact, at the GUT level there is just one (unified) gauge group, such that universality is trivial.

\section{The Hilbert superpotential bases for models with $\mathbb{Z}_{12}^{R}$ symmetries}

In Section 3.2, we discuss several $\mathbb{Z}_{M}^{R}$ symmetries that forbid neutrino masses perturbatively and also present the Hilbert superpotential basis for a model with a $\mathbb{Z}_{8}^{R}$ symmetry and two $\mathbb{Z}_{4}^{R}$ symmetries amended by an extra $\mathbb{Z}_{4}$ factor. In this appendix we provide further examples based on the $\mathbb{Z}_{12}^{R}$ symmetries. As we have already stated above, every possible superpotential term $\mathscr{M}$ contains only one inhomogeneous monomial and an arbitrary combination of homogeneous monomials [34, i.e.

$$
\mathscr{M}=\mathscr{M}_{\mathrm{in}}^{(i)} \prod_{j=1}\left(\mathscr{M}_{\mathrm{hom}}^{(j)}\right)^{\eta_{j}} \quad \text { with } \quad \eta_{j} \in \mathbb{N}
$$

where $\mathscr{M}_{\text {in }}^{(i)}$ is an inhomogeneous and $\mathscr{M}_{\text {hom }}^{(j)}$ a homogeneous monomial.

In Section 3.2 we list three examples which have a $\mathbb{Z}_{12}^{R}$ symmetry. As we will see in the following, the three sets of monomials differ. Hence, the three $\mathbb{Z}_{12}^{R}$ symmetries are inequivalent. The first symmetry has the charge assignment

$$
\left(\begin{array}{lllllll}
q_{10} & q_{\overline{\mathbf{5}}} & q_{H_{u}} & q_{H_{d}} & q_{\theta} & \rho & q_{\bar{\nu}}
\end{array}\right)=\left(\begin{array}{lllllll}
1 & 9 & 4 & 8 & 3 & 3 & 11
\end{array}\right),
$$

which leads to the inhomogeneous monomials

$$
\begin{aligned}
& \left(L H_{d} \bar{E}\right) ;\left(L H_{u}\right)^{6} ; \bar{\nu}^{6} ;(L L \bar{E}) \bar{\nu} ; \\
& (L L \bar{E})^{6} ;(L L \bar{E})^{4}\left(L H_{u}\right)^{2} ;(L L \bar{E})^{2}\left(L H_{u}\right)^{4},
\end{aligned}
$$

whereas the homogeneous ones are given by

$$
\begin{aligned}
& (L L \bar{E})^{12} ;\left(L H_{u}\right)^{12} ; H_{u} H_{d} ;\left(L H_{u}\right) \bar{\nu} ;(L L \bar{E})\left(L H_{d} \bar{E}\right) \bar{\nu} ; \\
& \bar{\nu}^{12} ;\left(L H_{d} \bar{E}\right)\left(L H_{u}\right)^{6} ;\left(L H_{d} \bar{E}\right)^{2} ;(L L \bar{E})^{7} \bar{\nu} ; \\
& (L L \bar{E})\left(L H_{u}\right)^{5} ;(L L \bar{E})^{6}\left(L H_{d} \bar{E}\right) ;\left(L H_{d} \bar{E}\right) \bar{\nu}^{6} ; \\
& (L L \bar{E})^{2} \bar{\nu}^{2} ;\left(L H_{d} \bar{E}\right)(L L \bar{E})^{4}\left(L H_{u}\right)^{2} ;(L L \bar{E})^{5}\left(L H_{u}\right) ; \\
& \left(L H_{d} \bar{E}\right)(L L \bar{E})^{2}\left(L H_{u}\right)^{4} ;(L L \bar{E}) \bar{\nu}^{7} ;(L L \bar{E})^{3}\left(L H_{u}\right)^{3} .
\end{aligned}
$$


The second $\mathbb{Z}_{12}^{R}$ symmetry has the charges

$$
\left(\begin{array}{lllllll}
q_{10} & q_{\overline{\mathbf{5}}} & q_{H_{u}} & q_{H_{d}} & q_{\theta} & \rho & q_{\bar{\nu}}
\end{array}\right)=\left(\begin{array}{lllllll}
2 & 6 & 2 & 10 & 3 & 3 & 4
\end{array}\right),
$$

which gives us for the inhomogeneous monomials

$$
\left(L H_{d} \bar{E}\right) ;(L L \bar{E})^{3} ;(L L \bar{E}) \bar{\nu} ;(L L \bar{E})\left(L H_{u}\right)^{2}
$$

and for the homogeneous monomials

$$
\begin{aligned}
& (L L \bar{E})^{6} ;(L L \bar{E})^{4} \bar{\nu} ;\left(L H_{d} \bar{E}\right)(L L \bar{E})^{3} ; H_{u} H_{d} ; \\
& \left(L H_{u}\right) \bar{\nu} ; \bar{\nu}^{3} ;\left(L H_{u}\right)^{3} ;\left(L H_{d} \bar{E}\right)\left(L H_{u}\right)^{2}(L L \bar{E}) ; \\
& (L L \bar{E})^{2}\left(L H_{u}\right) ;\left(L H_{d} \bar{E}\right)^{2} ;(L L \bar{E})^{2} \bar{\nu}^{2} ;\left(L H_{d} \bar{E}\right)(L L \bar{E}) \bar{\nu} .
\end{aligned}
$$

The last $\mathbb{Z}_{12}^{R}$ symmetry has

$$
\left(\begin{array}{lllllll}
q_{10} & q_{\overline{5}} & q_{H_{u}} & q_{H_{d}} & q_{\theta} & \rho & q_{\bar{\nu}}
\end{array}\right)=\left(\begin{array}{lllllll}
4 & 0 & 10 & 2 & 3 & 3 & 2
\end{array}\right),
$$

as its charge assignment, with these we get the inhomogeneous monomials

$$
\bar{\nu}^{3} ;\left(L H_{d} \bar{E}\right) ;(L L \bar{E}) \bar{\nu} ;\left(L H_{u}\right)^{3} ;(L L \bar{E})^{2}\left(L H_{u}\right),
$$

and the following homogeneous ones

$$
\begin{aligned}
& \left(L H_{u}\right)^{6} ;\left(L H_{d} \bar{E}\right)\left(L H_{u}\right)^{3} ; H_{u} H_{d} ;\left(L H_{d} \bar{E}\right)(L L \bar{E})^{2}\left(L H_{u}\right) ; \\
& \left(L H_{u}\right) \bar{\nu} ;(L L \bar{E})^{3} ;(L L \bar{E})\left(L H_{u}\right)^{2} ;\left(L H_{d} \bar{E}\right)^{2} ; \\
& \left(L H_{d} \bar{E}\right) \bar{\nu}^{3} ;\left(L H_{d} \bar{E}\right)(L L \bar{E}) \bar{\nu} ;(L L \bar{E}) \bar{\nu}^{4} ; \bar{\nu}^{6} ; \\
& (L L \bar{E})^{2} \bar{\nu}^{2} .
\end{aligned}
$$

\section{References}

[1] S. Dimopoulos, S. Raby, and F. Wilczek, Phys. Rev. D24 (1981), 1681.

[2] G. R. Farrar and P. Fayet, Phys. Lett. B76 (1978), 575.

[3] S. Dimopoulos, S. Raby, and F. Wilczek, Phys. Lett. B112 (1982), 133.

[4] N. Sakai and T. Yanagida, Nucl. Phys. B197 (1982), 533.

[5] S. Weinberg, Phys. Rev. D26 (1982), 287.

[6] I. Hinchliffe and T. Kaeding, Phys. Rev. D47 (1993), 279.

[7] G. F. Giudice and A. Masiero, Phys. Lett. B206 (1988), 480.

[8] H. M. Lee, S. Raby, M. Ratz, G. G. Ross, R. Schieren, K. Schmidt-Hoberg, and P. K. Vaudrevange, Nucl.Phys. B850 (2011), 1, arXiv:1102.3595 [hep-ph]. 
[9] L. J. Hall, Y. Nomura, and A. Pierce, Phys. Lett. B538 (2002), 359, hep$\mathrm{ph} / 0204062$.

[10] A. H. Chamseddine and H. K. Dreiner, Nucl.Phys. B458 (1996), 65, arXiv:hepph/9504337 [hep-ph].

[11] T. Banks and N. Seiberg, Phys.Rev. D83 (2011), 084019, arXiv: 1011.5120 [hepth].

[12] F. Brümmer, R. Kappl, M. Ratz, and K. Schmidt-Hoberg, JHEP 04 (2010), 006, arXiv:1003.0084 [hep-ph].

[13] J. E. Kim and H. P. Nilles, Phys. Lett. B138 (1984), 150.

[14] M. B. Green and J. H. Schwarz, Phys. Lett. B149 (1984), 117.

[15] G. 't Hooft, C. Itzykson, A. Jaffe, e. Lehmann, H., P. Mitter, et al., NATO Adv.Study Inst.Ser.B Phys. 59 (1980), 1.

[16] C. Csáki and H. Murayama, Nucl. Phys. B515 (1998), 114, hep-th/9710105.

[17] M. Fallbacher, M. Ratz, and P. K. Vaudrevange, Phys.Lett. B705 (2011), 503, arXiv:1109.4797 [hep-ph].

[18] H. P. Nilles, M. Ratz, and P. K. Vaudrevange, arXiv:1204.2206 [hep-ph].

[19] M. Dine and J. Kehayias, Phys.Rev. D82 (2010), 055014, arXiv:0909.1615 [hep$\mathrm{ph}]$.

[20] K. S. Babu, I. Gogoladze, and K. Wang, Nucl. Phys. B660 (2003), 322, hep$\mathrm{ph} / 0212245$.

[21] H. M. Lee, S. Raby, M. Ratz, G. G. Ross, R. Schieren, K. Schmidt-Hoberg, and P. K. Vaudrevange, Phys.Lett. B694 (2011), 491, arXiv:1009.0905 [hep-ph].

[22] E. Witten, Nucl. Phys. B188 (1981), 513.

[23] M. Dine, J. L. Feng, and E. Silverstein, Phys. Rev. D74 (2006), 095012, hepth/0608159.

[24] H. P. Nilles, Phys. Lett. B115 (1982), 193.

[25] K. Intriligator, N. Seiberg, and D. Shih, JHEP 07 (2007), 017, hep-th/0703281.

[26] N. Arkani-Hamed, L. J. Hall, H. Murayama, D. Tucker-Smith, and N. Weiner, Phys.Rev. D64 (2001), 115011, arXiv:hep-ph/0006312 [hep-ph].

[27] F. Borzumati and Y. Nomura, Phys.Rev. D64 (2001), 053005, arXiv:hepph/0007018 [hep-ph]. 
[28] J. March-Russell and S. M. West, Phys.Lett. B593 (2004), 181, arXiv:hep$\mathrm{ph} / 0403067$ [hep-ph].

[29] J. L. Evans, M. Ibe, J. Kehayias, and T. T. Yanagida, arXiv:1111.2481 [hep-ph].

[30] R. N. Mohapatra and M. Ratz, Phys. Rev. D76 (2007), 095003, arXiv: 0707.4070 [hep-ph].

[31] R. Kappl, B. Petersen, S. Raby, M. Ratz, R. Schieren, and P. K. Vaudrevange, Nucl.Phys. B847 (2011), 325, arXiv: 1012.4574 [hep-th].

[32] B. Petersen, M. Ratz, and R. Schieren, JHEP 08 (2009), 111, arXiv:0907.4049 [hep-ph].

[33] C. Luhn and M. Thormeier, Phys.Rev. D77 (2008), 056002, arXiv: 0711.0756 [hep$\mathrm{ph}]$.

[34] R. Kappl, M. Ratz, and C. Staudt, JHEP 1110 (2011), 027, arXiv:1108.2154 [hep-th].

[35] M. Cvetič, L. L. Everett, and J. Wang, Phys. Rev. D59 (1999), 107901, hep$\mathrm{ph} / 9808321$.

[36] K. Fujikawa, Phys. Rev. Lett. 42 (1979), 1195.

[37] K. Fujikawa, Phys. Rev. D21 (1980), 2848.

[38] C. Lüdeling, F. Ruehle, and C. Wieck, Phys.Rev. D85 (2012), 106010, arXiv:1203.5789 [hep-th].

[39] K. Huitu, Y. Kawamura, T. Kobayashi, and K. Puolamaki, Phys. Rev. D61 (2000), 035001, hep-ph/9903528.

[40] A. Hebecker and M. Trapletti, Nucl. Phys. B713 (2005), 173, hep-th/0411131.

[41] M. Trapletti, Mod.Phys.Lett. A21 (2006), 2251, arXiv:hep-th/0611030 [hep-th].

[42] A. Anandakrishnan and S. Raby, arXiv:1205.1228 [hep-ph].

[43] R. Blumenhagen, G. Honecker, and T. Weigand, JHEP 06 (2005), 020, hepth/0504232.

[44] S. Groot Nibbelink, H. P. Nilles, and M. Trapletti, Phys. Lett. B652 (2007), 124, hep-th/0703211. 